\title{
DR-10
}

\section{BRØNSTED ACIDIC IONIC LIQUID-CATALYZED ONE-POT SYNTHESIS OF 4(3H)QUINAZOLINONES UNDER SOLVENT-FREE CONDITIONS}

\author{
Subhankar Sarkar, ${ }^{1}$ Rana Chatterjee, ${ }^{1}$ Sougata Santra, ${ }^{2}$ Grigory V. Zyryanov, ${ }^{2,3}$ Adinath Majee \\ ${ }^{1}$ Department of Chemistry; Visva-Bharati (A Central University), Santiniketan 731235, India. \\ ${ }^{2}$ Ural Federal University, 19 Mira St., Yekaterinburg, 620002, Russian Federation. \\ ${ }^{3}$ Ya. Postovsky Institute of Organic Synthesis UB RAS, S. Kovalevskoy/Akademicheskaya St., 20/22, \\ Yekaterinburg,620990, Russian Federation. E-mail: sarkarsubhankar93@ gmail.com
}

\begin{abstract}
Butane sulfonic acid-3-methylimidazolium tosylate, [BSMIM]OTs, is found to be a remarkable catalyst for the synthesis of $4(3 H)$-quinazolinones undersolvent-free conditions. This developed synthetic procedureis applicable for the construction of $4(3 H)$-quinazolinone and its derivatives.These $4(3 H)$-quinazolinone systems have a wide range of useful biological properties, such as anticancer, antiviral, anti-inflammatory, anti-microbial cholinesterase inhibitor, antifolate, antitumor, protein kinase inhibitor and many others. ${ }^{1}$ Some of these of quinazolinone derivatives also show potentially pharmacological activities. ${ }^{2}$ That's way from long year, many methods have been developed for the synthesis of 4(3H)-quinazolinonesusing various catalytic reagents. ${ }^{3}$ Keeping in mind the applicability of BAILs we have developed a mild and green synthetic protocol which explore the synthesis of $4(3 H)$ quinazolinones compounds. The feasibility of catalyst recycling has also been demonstrated. Clean reaction, short reaction time, easily accessible reactants, and metal and solvent-free and environmentally friendly reaction conditions are the notable advantages of this procedure (Scheme 1).
\end{abstract}

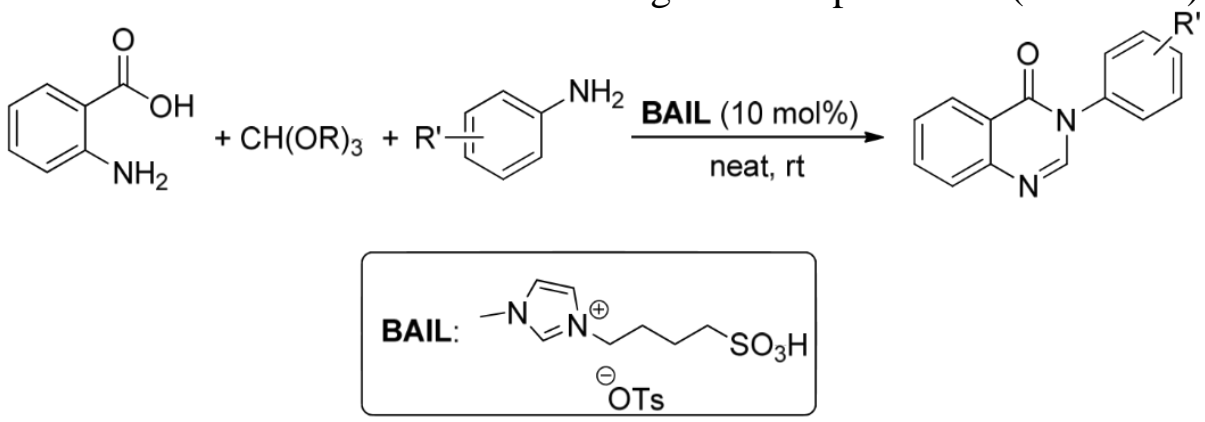

Scheme 1. Synthesis of 4(3H)-quinazolinones using [BSMIM]OTs

\section{References}

1. Quinoline, quinazoline and acridone alkaloids / J. P. Michael // Nat. Prod. Rep.- 2004. - Vol.21. - P. 650-668.

2. Quinazolinone: an overview / R. Arora, A. Kapoor, N. S. Gill, A. C. Rana // Int. Res. J. Pharm. - 2011. - Vol. 2. - P. 2228.

3. $\mathrm{Bi}(\mathrm{TFA})_{3}-[\mathrm{nbp}] \mathrm{FeCl}_{4}$ : a new, efficient and reusable promoter system for the synthesis of $4(3 \mathrm{H})$ quinazolinone derivatives / A. R. Khosropour, I. Mohammadpoor-Baltork, H. Ghorbankhani // Tetrahedron Lett. - 2006. - Vol. 47. - P. $3561-3564$.

This work was developed by Russian Science Foundation (Ref. No. 18-73-00301). 